Research Article

\title{
Numerical Simulation and Experiments on Muzzle Blast Overpressure in Large- Caliber Weapons
}

\author{
Lei Hong-xia ${ }^{1{ }^{1 *},}$ Zhao Jun-li ${ }^{1}$, and Wang Zhi-jun ${ }^{1}$

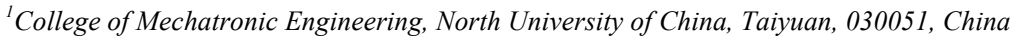

Received 28 July 2016; Accepted 29 October 2016

\begin{abstract}
A blast wave, especially from muzzle-loaded weapons, actually causes serious harm to gunners and weapons, as well as the surrounding environment. Therefore, accurate evaluation of high-pressure blast wave is considered a critical issue. The blast overpressure produced by muzzle blast wave in large-caliber weapons was performed numerically and experimentally in this study to resolve the problem above. First, two 3D computational models were constructed: one with a precursor flow field and the other without one. The muzzle flow field with a complicated projectile was simulated numerically. A second-order upwind scheme and multi-domain structured grids were employed to solve Navier-Stokes governing equations, and the Spalart-Allmaras model was utilized for turbulent flow calculation. Second, the muzzle blast overpressure over time in these two cases was calculated thoroughly. Finally, the values of the muzzle blast overpressure at different key points were tested by experiments. Results show that the peak overpressure in the area near the muzzle is larger when the precursor flow is considered. Moreover, the simulation values with the precursor flow are closer to the experimental values than those without the precursor flow. When the tested point is closer to the muzzle, its overpressure is affected greater by the precursor flow. Thus, precursor flow should be considered in the design of the muzzle device but should be neglected in the analysis of the far field. This study is meaningful and significant in studying the process of muzzle shockwave and in designing muzzle devices.
\end{abstract}

Keywords: Muzzle blast overpressure, Dynamic mesh, Unsteady flow, Precursor flow.

\section{Introduction}

A projectile is accelerated by the abrupt explosion of the propellant inside the gun barrel during a gun firing. Simultaneously, the precursor shockwave is formed ahead of the projectile. Another blast wave is formed after the projectile leaves the muzzle, which is produced by the highpressure propellant gas behind the projectile [1,2]. All of these waves can produce various complex physical coupling phenomena, such as the muzzle flash near the muzzle, muzzle blast wave, and noise in the far field. These physical phenomena bring different effects, such as damage to human bodies and equipment around the gun, and greatly reduce artillery performance. The least serious result is a short period of tinnitus and deafness, and serious consequences can be earache, ear bleeding, and even internal hemorrhage to the gunner. These phenomena are more obvious and destructive for large-caliber guns. Thus, the muzzle shockwave directly affects the health of the gunner.

These kinds of muzzle blast flow usually have many special features and properties, such as high energy, impulsiveness, low frequency, strong directivity, and longrange propagation [3]. The muzzle blast wave is highly directional that sound effect at the locations directly in front of the gun is about $15 \mathrm{~dB}$ higher than in equidistant locations directly to the rear of the gun [4]. According to some experimental investigations, the noise levels due to highpressure blast flow could be heard about 10 miles away from the firing point at a level of $90 \mathrm{~dB}$ [3]. The modern war has

*E-mail address: Leihongxia@nuc.edu.cn

ISSN: $1791-2377$ (C) 2016 Eastern Macedonia and Thrace Institute of Technology. All rights reserved. set higher requirements for the comprehensive performance of artilleries. For example, guns should have more power and maneuverability, and must secure the safety of the operator and equipment. Exploring the muzzle shock wave attenuation rule is important in the design of modern artillery to reduce or eliminate the adverse effects mentioned previously. Therefore, the accurate simulation and measurement of the muzzle blast wave are becoming important parts of the gun system during research.

\section{State of the Art}

Many studies on muzzle blast flows have been conducted numerically with the recent development of computational fluid dynamics (CFD) technology [5, 6, 7, 8, 9]. These studies have provided considerable insight into blast wave structure and propagation. However, the movement and the complex geometry of the projectile were ignored or simplified. To study the detailed structures of the blast flow, Chevaugeon et al. [10] used discontinuous Galerkin methods and mesh adaptation methods to solve the 2D compressible Euler equations, and predicted shock strengths and blast overpressure. Zhang et al. [11] simulated the flying away of a high-speed projectile from the bores through different muzzle brakes using a high-resolution Roe scheme and structured dynamic mesh techniques based on the 2D unsteady Euler equations. As for muzzle device investigations, Florio [12] studied the effects of auxiliary flow tube vent opening area and arrangement on the gas flow field as a gas propelled cylinder exited a flow tube, and the researcher found that larger single openings were more 
effective in reducing the end of flow tube pressure, whereas multiple openings were more conducive to a reduced recoil force. Hudson and Luchini [13] evaluated the utility of computational models in the design of suppressors for smallcaliber guns. The projectile in their study was of the real shape, however, the 2D calculation model, especially with muzzle devices, could not accurately reflect the developing process of the flow field. Moreover, the research was performed based on small-caliber weapons and not on largecaliber weapons.

Many experiments have also been performed by several researchers [14, 15, 16]. However, these experiments were performed based on small weapons, and focused on the structure of the flow field and the pressure produced by the muzzle blast of different muzzles. The overpressure influenced by the precursor flow, especially for large-caliber weapons, has not been reported in the corresponding literature. The overpressure is essential in the research on vehicle-mounted guns to achieve the reasonable design of car body structure.

The present study focuses on a large-caliber muzzleloaded gun. The real shape of the projectile and moving-grid methods are considered in simulations. Moreover, 3D Navier-Stokes $(\mathrm{N}-\mathrm{S})$ equations are used for the governing equations to evaluate the overpressure of the muzzle blast overpressure accurately. In this way, the complex structure of the muzzle flow can be reflected clearly, and the peak overpressure is closer to the actual value.

The remaining part of this paper is organized as follows: Section 3 describes the methodology used in this study. Section 4 presents the numerical analyses in detail by constructing two 3D computational models and testing the muzzle blast wave overpressure at given points in largecaliber weapons. Finally, the conclusions are summarized in Section 5.

\section{Methodology}

\subsection{Governing equations}

The gunpowder gas is regarded as the perfect gas and ignores the chemical reaction to simulate the complex flow through the muzzle brake. Three-dimensional viscous and compressible $\mathrm{N}-\mathrm{S}$ equations can be described by the following equations:

$$
\frac{\partial U}{\partial t}+\frac{\partial F}{\partial x}+\frac{\partial G}{\partial y}+\frac{\partial H}{\partial z}=\frac{\partial F_{1}}{\partial x}+\frac{\partial G_{1}}{\partial y}+\frac{\partial H_{1}}{\partial z}
$$

where $U$ is the variable to be solved. $F, G$, and $H$ are the inviscid fluxes in three coordinate directions. $F_{1}, G_{1}$, and $H_{1}$ are the viscid fluxes in three coordinate directions. And $U$, $F, G, H, F_{1}, G_{1}$, and $H_{1}$ are defined, respectively, as follows:

$$
U=\left[\begin{array}{l}
\rho \\
\rho u \\
\rho v \\
\rho w \\
e
\end{array}\right], \quad F=\left[\begin{array}{l}
\rho u \\
\rho u^{2}+p \\
\rho u v \\
\rho w u \\
(e+p) u
\end{array}\right], G=\left[\begin{array}{l}
\rho v \\
\rho u v \\
\rho v^{2}+p \\
\rho v w \\
(e+p) v
\end{array}\right]
$$

$H=\left[\begin{array}{l}\rho w \\ \rho w u \\ \rho w v \\ \rho w^{2}+p \\ (e+p)_{w}\end{array}\right], F_{1}=\left[\begin{array}{l}0 \\ \tau_{x x} \\ \tau_{x y} \\ \tau_{x z} \\ u \tau_{x x}+v \tau_{x y}+w \tau_{x z}-q_{x}\end{array}\right]$,

$G_{1}=\left[\begin{array}{l}0 \\ \tau_{y x} \\ \tau_{y y} \\ \tau_{y z} \\ u \tau_{y x}+v \tau_{y y}+w \tau_{y z}-q_{y}\end{array}\right], H_{1}=\left[\begin{array}{l}0 \\ \tau_{z x} \\ \tau_{z y} \\ \tau_{z z} \\ u \tau_{z x}+v \tau_{z y}+w \tau_{z z}-q_{z}\end{array}\right]$,

$\tau_{x x}=\frac{2}{3} \mu\left(2 \frac{\partial u}{\partial x}-\frac{\partial v}{\partial y}-\frac{\partial w}{\partial z}\right), \tau_{x y}=\tau_{y x}=\mu\left(\frac{\partial u}{\partial y}+\frac{\partial v}{\partial x}\right)$,

$\tau_{y y}=\frac{2}{3} \mu\left(2 \frac{\partial v}{\partial y}-\frac{\partial u}{\partial x}-\frac{\partial w}{\partial z}\right), \quad \tau_{x z}=\tau_{z x}=\mu\left(\frac{\partial w}{\partial x}+\frac{\partial u}{\partial z}\right)$

$\tau_{z z}=\frac{2}{3} \mu\left(2 \frac{\partial w}{\partial z}-\frac{\partial u}{\partial x}-\frac{\partial v}{\partial y}\right), \tau_{y z}=\tau_{z y}=\mu\left(\frac{\partial v}{\partial z}+\frac{\partial w}{\partial y}\right)$

$q_{x}=-k \frac{\partial T}{\partial x}, q_{y}=-k \frac{\partial T}{\partial y}, \quad q_{z}=-k \frac{\partial T}{\partial z}$,

where $\rho$ is density; $u, v$, and $w$ are Cartesian velocity components for the $x$-, $y$-, and $z$-directions, respectively; $p$ is pressure; $k$ is gas conductivity; $\boldsymbol{\tau}_{i j}$ represents the viscous stresses; and $e$ is the total energy per unit volume which is defined as:

$e=\frac{p}{\gamma-1}+\frac{1}{2} \rho\left(u^{2}+v^{2}+w^{2}\right)$

Parameter $\gamma$ is the specific heat ratio. Ideal gas equation of state is defined as:

$$
p=\rho R T
$$

Prandtl number is expressed as:

$P_{r}=\frac{c_{p}}{k} \mu \approx 0.72$

and $\mu$ is given by

$$
\mu=C_{1} \frac{T^{3 / 2}}{T+C_{2}}
$$

Eqs. (1) to (5) constitute a closed system of equations.

\subsection{Turbulence model}

The Spalart-Allmaras model is applied for the turbulent flow calculation. This model includes a developed singleequation model and is effective for simulating boundarylayer flow fields for adverse pressure gradient problems. The transport equation for turbulent energy of the SpalartAllmaras model is expressed as follows: 


$$
\frac{\partial(\rho k)}{\partial t}+\frac{\partial\left(\rho k u_{i}\right)}{\partial x_{i}}=\frac{\partial}{\partial x_{j}}\left[\left(\mu+\frac{u_{i}}{\sigma_{k}}\right) \frac{\partial k}{\partial x_{j}}\right]+\mu_{t}\left(\frac{\partial u_{i}}{\partial x_{j}}+\frac{\partial u_{j}}{\partial x_{i}}\right) \frac{\partial u_{i}}{\partial x_{j}}-\rho C_{D} \frac{k^{\frac{2}{3}}}{l}
$$

From left to right, the terms account for transient, advection, diffusion, generation, and dissipation effects, respectively. The turbulent viscosity is calculated by the Kolmogorov-Prandtl expression:

$\mu_{t}=\rho C_{\mu} \sqrt{k} l$

$\sigma_{k}, C_{D}, C_{\mu}$ are empirical coefficients.

\subsection{Numerical analysis method}

A stable converged result is calculated in every time step for unsteady problems. In this work, finite volume method is used for space discretization. The second-order upwind scheme is adopted to discrete flux, and the explicit two-step Runge-Kutta method is used in time marching.

\section{Simulation Analysis}

\subsection{Computational domain}

The large-caliber $122-\mathrm{mm}$ gun is selected to analyze the muzzle blast wave. A quarter of the axisymmetric computational domain is constructed, which is shown in Fig. 1. Two models are set up to study the overpressure produced by the muzzle blast wave. Model 1 is set up without initial muzzle flow, including a projectile moving from the muzzle. Model 2 is set up with the initial flow field and the projectile moving from the bottom of the tube according to internal ballistics.

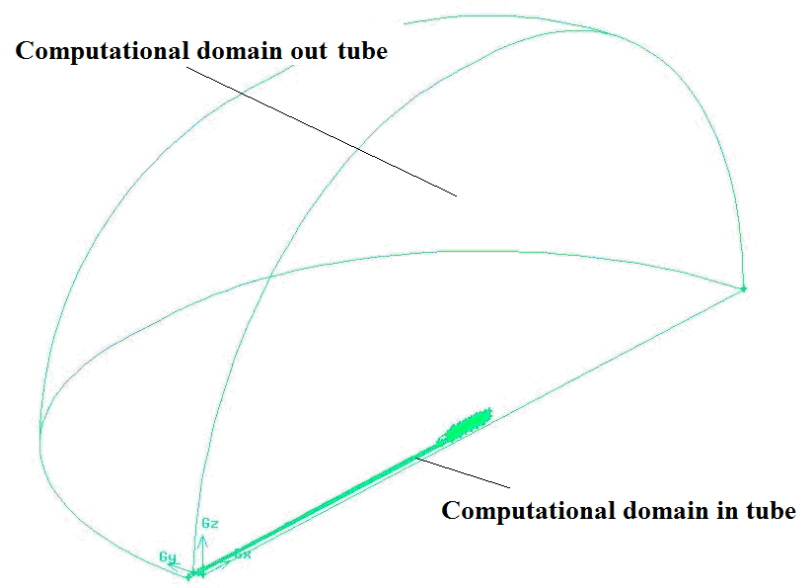

Fig. 1. Computational domain for the 122-mm gun

\subsection{Mesh methods}

Multi-domain structured grids are employed in the simulations. Hybrid structured grid and moving-grid methods are used in the internal ballistic period. Quadrilateral mesh is applied for the regular surfaces. The hexahedral mesh is applied for the area far from the muzzle. Adaptive mesh refinement is applied to the numerical simulation of the muzzle flow around the projectile. Mesh diagram is shown in Fig. 2.

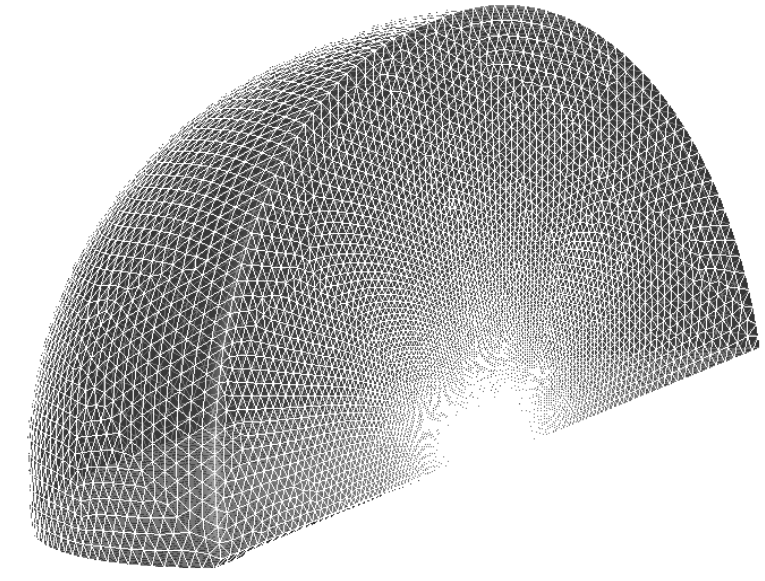

Fig. 2. Mesh diagram for the 122-mm gun

\subsection{Initial and boundary conditions}

Initial conditions include velocity, pressure, density, and temperature. In the period of internal ballistics, the friction between the projectile and the tube is negligible, and the driving force acting on the projectile is sufficient enough to overcome the friction and the drag force to keep the projectile moving completely inside the tube. Projectile motion follows Newton's law when the projectile begins to move out of the tube. The loading conditions include pressure, velocity, and temperature of the gunpowder gases in the tube. These parameter distributions can be obtained by solving the internal ballistics.

The boundary conditions used in this simulation include plane-symmetric, moving, wall, and pressure outlet boundaries. Plane-symmetric and pressure outlet boundaries are shown in Fig. 3.

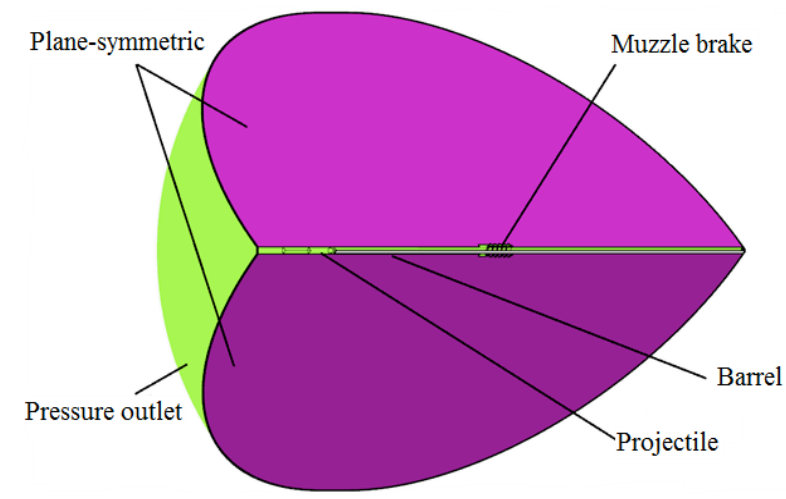

Fig. 3. Boundary conditions

\subsection{Muzzle blast pressure analysis}

Different points are selected at a radial distance of $0.88 \mathrm{~m}$, $1.28 \mathrm{~m}$, and $1.67 \mathrm{~m}$ from the center of the muzzle brake to obtain the overpressure produced by muzzle blast wave under the muzzle brake. These points have been taken at an angle of $0^{\circ}, 30^{\circ}$, and $45^{\circ}$, respectively, as shown in Fig. 4. 


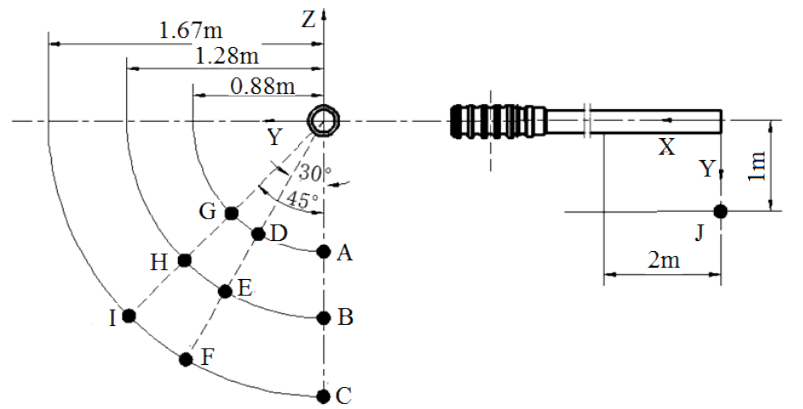

Fig. 4. Schematic diagram for monitoring points

Fig. 5 shows the $\mathrm{p}-\mathrm{t}$ curves of the given points with the same direction. As shown in the figure, the numerical simulation results of the two models are similar. The values of the model with precursor flow field are higher than those of the model without precursor flow field. The overpressure values at the given points are almost zero in the period of internal ballistics. However, the overpressure values at the given points increase rapidly in a very short period of time and reach their maximum values within $1 \mathrm{~ms}$ as the highpressure propellant gas begins to flow out of the side holes of the muzzle at the beginning of the after-effect period. As shown in Fig. 5, the pressure value at point $G$ is up to its maximum initially in the $30^{\circ}$ direction. The same phenomenon takes place at points $\mathrm{H}$ and $\mathrm{I}$, respectively. The pressure value is maximized at point $\mathrm{G}$ at the peripheral direction, which is $0.88 \mathrm{~m}$ away from the gun muzzle, followed by points D and A. However, as shown in Fig. 6, the calculation overpressure results of the two models are basically the same at the gunner position farther away from the muzzle. Thus, the influence of the precursor flow field is mainly at the beginning of the after-effect period, whereas the effect on the latter is insignificant.

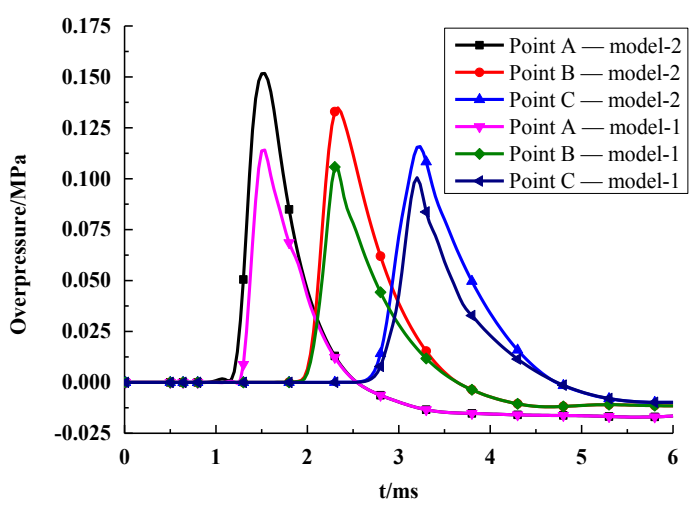

(a) $0^{\circ}$

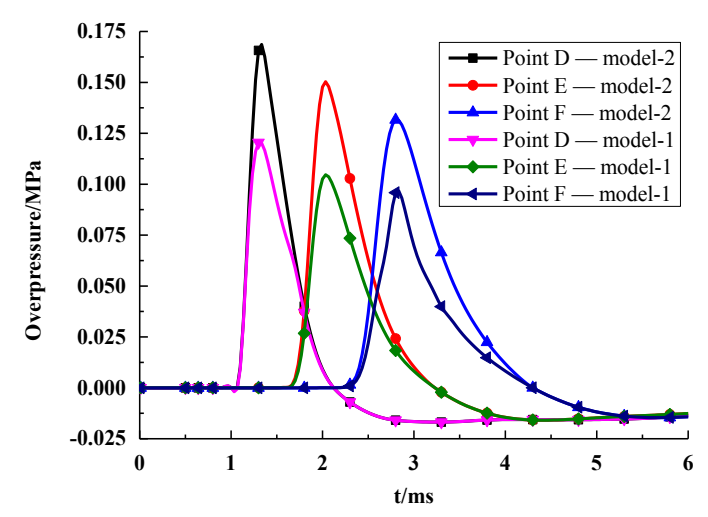

(b) $30^{\circ}$

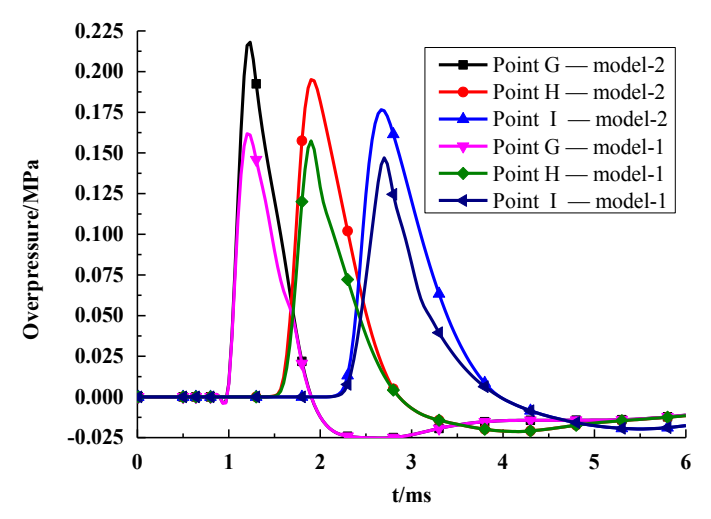

(c) $45^{\circ}$

Fig. 5. Overpressure at given points of different angles

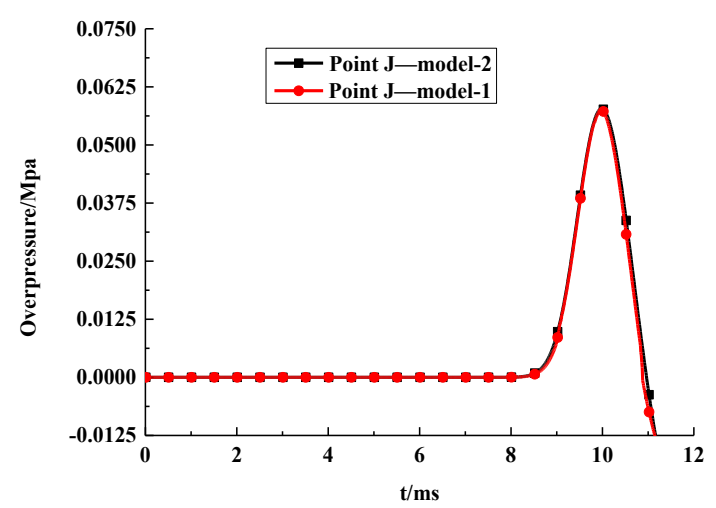

Fig. 6. Overpressure at the gunner position

\subsection{Living firing test}

A set of test system is designed to fix the sensor to test the change of shockwave at the given points below the muzzle brake and the breech position at the state of $0^{\circ}$ traversing angle and $30^{\circ}$ elevating angle. The schematic diagram of the test system is shown in Fig. 7, and the test scene is shown in Fig. 8.

Fig. 9 is the p-t curves of the test overpressure at points C, D, E, F, I, and J. The curves in the graph correctly reflect the variation law of shockwave propagation, and the test results capture the main shockwave maximum overpressure point. Simultaneously, fluctuation phenomenon can be found in the test results in the process of shockwave attenuation because of the existence of chemical reaction and the secondary muzzle flash in the real process of projectile firing.

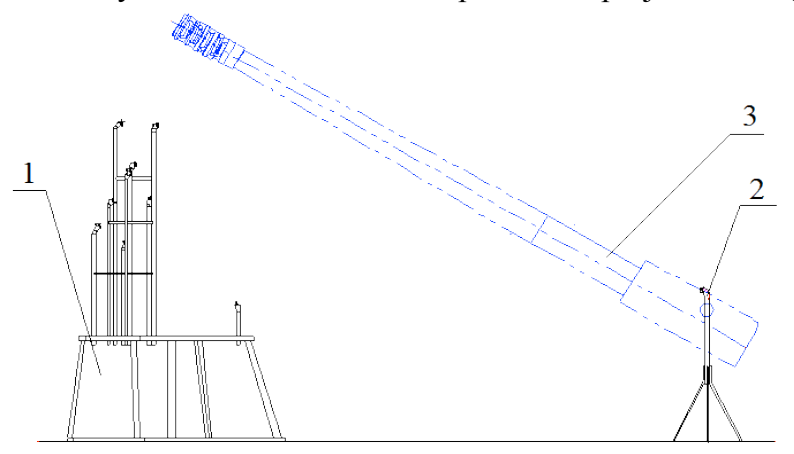

$\begin{array}{llll}\text { 1. Text support } 1 & 2 \text {. Test support } 2 \text { 3. Gun }\end{array}$

Fig. 7. Schematic diagram of the test system 


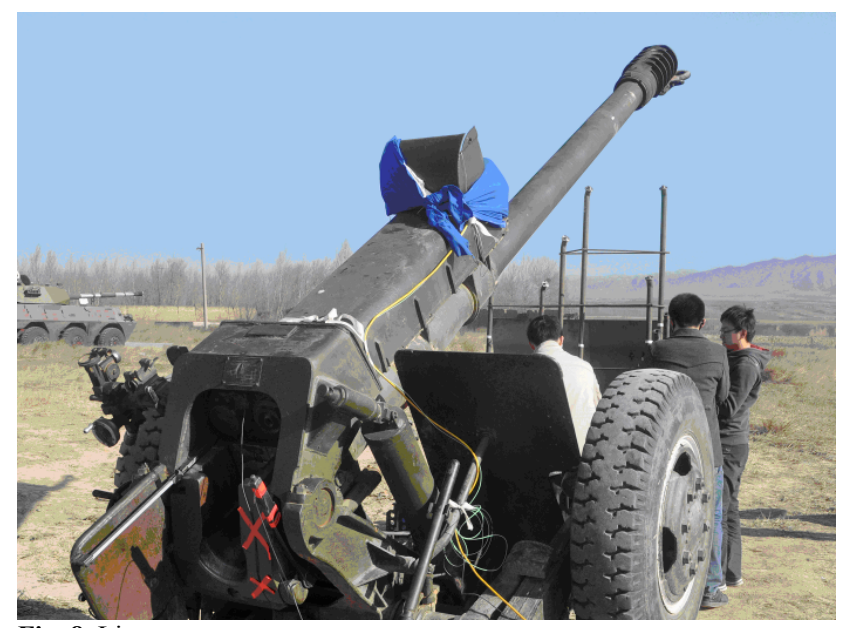

Fig. 8. Live test

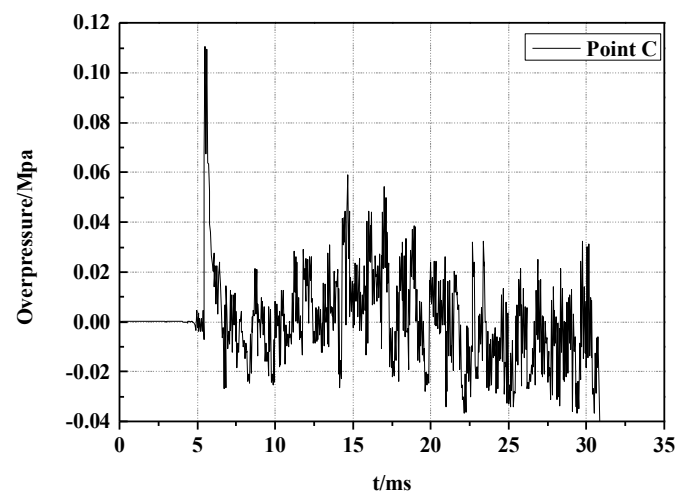

(a) Point C

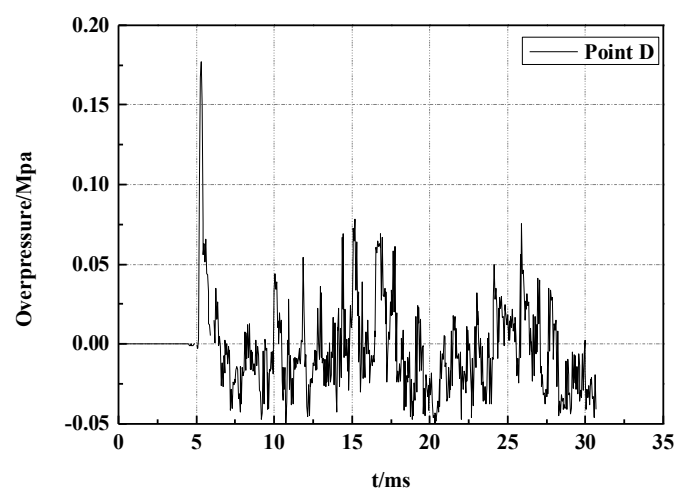

(b) Point D

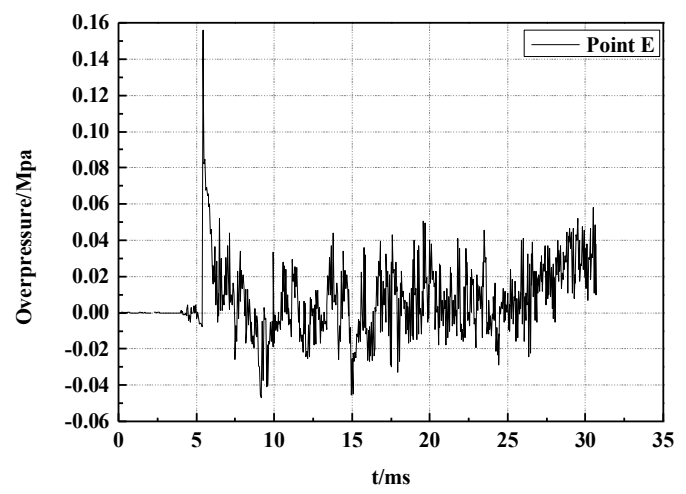

(c) Point E

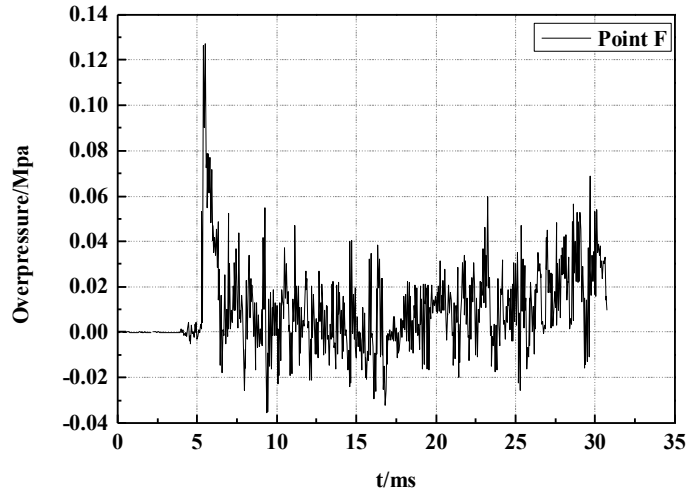

(d) Point F

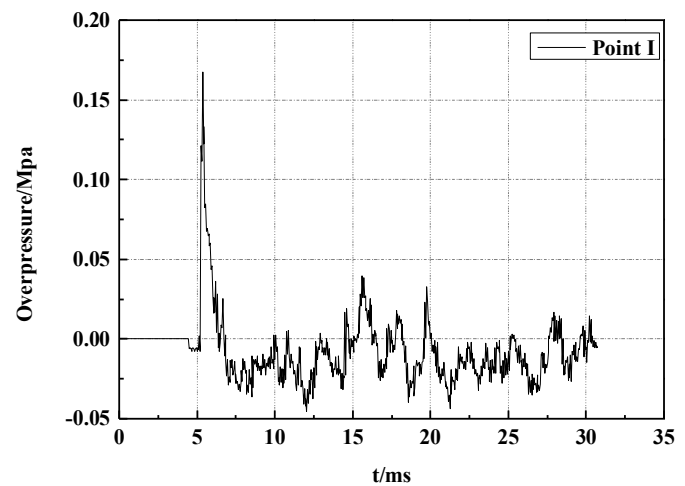

(e) Point I

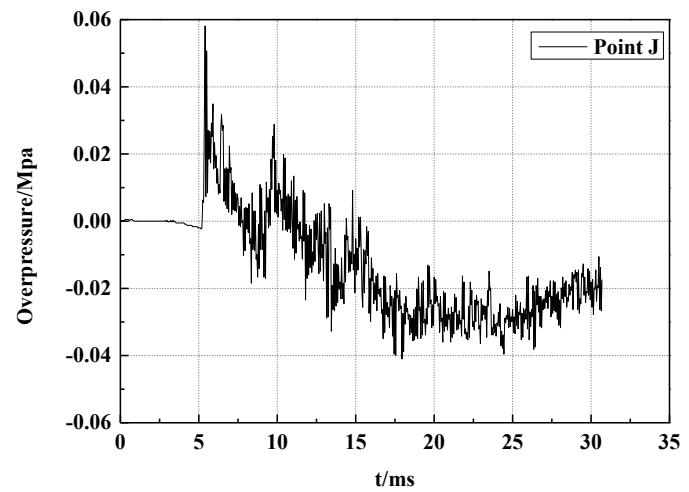

(f) Point J

Fig. 9. $\mathrm{P}-\mathrm{t}$ curves of the test overpressure at given points

Tab. 1 shows the peak overpressure at tested points. The simulation results of Model 2 are closer to the test data. The test values are slightly higher than the calculated values considering precursor flow field. However, the change rules of each monitored point are consistent with the numerical calculation, because the computing grid in the flow field is not sufficiently thin. The calculating time increases with the number of computing grids. Therefore, the appropriate grid size and quantity should be selected according to the actual situation.

Table 1 Peak overpressure at tested points

\begin{tabular}{c|c|c|c}
\hline \multirow{2}{*}{ Location } & \multicolumn{3}{|c}{ Peak overpressure/Mpa } \\
\cline { 2 - 4 } & Model 1 & Model 2 & Text \\
\hline Point C & 0.0905 & 0.1055 & 0.1106 \\
Point D & 0.1204 & 0.1657 & 0.1768 \\
Point E & 0.1126 & 0.1478 & 0.1562
\end{tabular}




\begin{tabular}{l|l|l|l} 
Point F & 0.1031 & 0.1216 & 0.1237 \\
Point I & 0.1470 & 0.1659 & 0.1676 \\
Point J & 0.0563 & 0.0569 & 0.0583 \\
\hline
\end{tabular}

\section{Conclusion}

This study aims to evaluate the muzzle blast overpressure accurately and explore the effects of the precursor flow on muzzle blast overpressure. Thus, numerical simulation of muzzle blast overpressure based on CFD technology was conducted. Then, the second-order upwind scheme was employed to solve $\mathrm{N}-\mathrm{S}$ governing equations. Finally, the simulated peak overpressure was verified by numerical experiments. The main conclusions are as follows:

(1) The numerical simulation results of the two models are similar. The values of the model with precursor flow field are higher than that of the model without precursor flow field. When the distance is farther away from the muzzle, the difference between the peak overpressure calculated by the two models is smaller. The calculation overpressure results are basically the same for the gunner position farther away from the muzzle.

(2) The peak overpressure values of the given points based on the model with precursor flow field agree well with the experimental values. The numerical simulation method considering the precursor flow field can be used to evaluate the shockwave overpressure, which can greatly reduce the test cost and shorten the design cycle for the weapon system.

(3) The influence of the precursor flow field is mainly at the beginning of the after-effect period, whereas the effect on the latter is small. Therefore, the precursor flow field can be neglected during the middle and far-field calculation of the shockwave. However, the initial flow field should be considered when designing muzzle devices.

This study is useful for the design of weapon system, particularly for the muzzle device. Nonetheless, the study is hindered by a number of limitations. The chemical reactions between the propellant gas and the influence of recoil were not considered in the analysis. In future works, the simulation results should be further studied.

\section{References}

1. Rajesh Gopalapillai, Heuy-Dong Kim, Toshiaki Setoguchi and Shigeru Matsuo, "On the near-field aerodynamics of a projectile launched from a ballistic range", journal of Mechanical Science and Technology, 21(7), 2007, pp. 1129-1138.

2. A. Merlen, "Generalization of the muzzle wave similarity rules", Shock Waves, 9(5), 1999, pp. 341-352.

3. Kuk-Jeong Kang, Sung-Ho Ko and Dong-Soo Lee, "A study on impulsive sound attenuation for a high-pressure blast flowfield", Journal of Mechanical Science and Technology, 22(1), 2008, pp. 190-200.

4. Hafizer Rehman, Seung Hwa Hwang, Berkah Fajar, Hanshik Chung and Hyomin Jeong, "Analysis and attenuation of impulsive sound pressure in large caliber weapon during muzzle blast", Journal of Mechanical Science and Technology, 25(10), 2011, pp. 2601-2606.

5. Zonglin Jiang, "Wave dynamic processes induced by a supersonic projectile discharging from a shock tube", Physics of Fluids, 15 (6), 2003, pp. 1665-1675.

6. Xiaohai Jiang, Zhihua Chen, Baochun Fan, Hongzhi Li, "Numerical simulation of blast flow fields induced by a high-speed projectile", Shock Waves, 18, 2008, pp. 205-212.

7. Jonghoon Bin, Minwoo Kim, Soogab Lee, "A numerical study on the generation of impulsive noise by complex flows discharging from a muzzle", International Journal for Numerical Methods in Engineering, 75 (8), 2008, pp. 964-991.

8. Y. Dayan and D Touati, "Simulation of unsteady muzzle flow of a small caliber gun", Advances in fluid mechanics VI, 2006, pp 165 171 .
9. D L Cler, N. Chevaugeon, MS Shephard and Jean-François Remacle, "CFD application to gun muzzle blast--a validation case study". In: Proceedings of 41st Aerospace Sciences Meeting and Exhibit, Reno, Nevada: AIAA, 2003, pp. 2003-1142.

10. N. Chevaugeon, J. Xin, P. Hu, X. Li, D. Cler, J.E. Flaherty and M.S. Shephard, "Discontinuous Galerkin Methods Applied to Shock and Blast Problems", Journal of Scientific Computing, 22(1), 2005, pp. 227-243.

11. Zhang Huan-hao, Chen Zhi-hua, Jiang Xiao-hai, HAN Jun-li, "Investigation on the Blast Wave Structures of a High-speed projectile flying through different muzzle brakes", Acta Armamentarii, 33 (5), 2012, pp. 23-629.

12. L. A. Florio, "Effect of vent opening area and arrangement on gas flow field as gas propelled cylinder exits a flow tube", Meccanica, 45(4), 2010, pp. 475-501.

13. M.KeithHudson and ChrisLuchini, "Evaluation of Computational Fluid Dynamics Methods for Design of Muzzle Blast Suppressors for Firearms", Propellants, Explosives, Pyrotechnics, 26, 2001, pp. 201-208

14. A. Merlen and A. Dyment, "Similarity and asymptotic analysis for gun-firing aerodynamics", Journal of Fluid Mechanics, 225, 1991, pp. 497-528.

15. M.M. Biss, G.S. Settles, M.J. Hargather, L.J. Dodson, and J.D. Miller, "High-speed digital shadowgraphy of shock waves from explosions and gunshots", Shock Waves, (2), 2009, pp. 91-96.

16. Toshiharu Mizukaki, "Detached shock waves around cylinders flying at mach number ranging from 1 to 2", Journal of Visualization, 11 (2), 2008, pp. 133-141. 